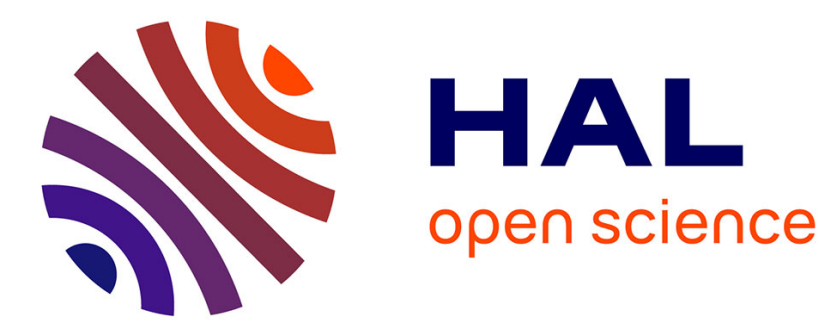

\title{
On Quasiconvex Functions Which are Convexifiable or Not \\ Jean-Pierre Crouzeix
}

\section{To cite this version:}

Jean-Pierre Crouzeix. On Quasiconvex Functions Which are Convexifiable or Not. Journal of Optimization Theory and Applications, 2021, 10.1007/s10957-021-01965-1 . hal-03432694

\section{HAL Id: hal-03432694 \\ https://hal.uca.fr/hal-03432694}

Submitted on 17 Nov 2021

HAL is a multi-disciplinary open access archive for the deposit and dissemination of scientific research documents, whether they are published or not. The documents may come from teaching and research institutions in France or abroad, or from public or private research centers.
L'archive ouverte pluridisciplinaire HAL, est destinée au dépôt et à la diffusion de documents scientifiques de niveau recherche, publiés ou non, émanant des établissements d'enseignement et de recherche français ou étrangers, des laboratoires publics ou privés. 
Abstract A quasiconvex function $f$ being given, does there exist an increasing and continuous function $k$ which makes $k \circ f$ convex? How to build such a $k$ ? Some words on least convex (concave) functions. The ratio of two positive numbers is neither locally convexifiable nor locally concavifiable. Finally, some considerations on the approximation of a preorder from a finite number of observations and on the revealed preference problem.

Communicated by Boris S. Mordukhovich

Keywords Convexifiability, Least Concavity, Preorder, Utility Functions, Afriat's and Sandwich Approximations, Revealed Preferences

Mathematics Subject Classification (2000) 91B42 $26 \mathrm{~B} 25$ 


\section{On Quasiconvex Functions Which Are Convexifiable Or}

Not

\section{Jean-Pierre Crouzeix}

dedicated to Franco Gianessi

Received: date / Accepted: date

\section{Introduction.}

A function $f: \mathrm{R}^{\mathrm{n}} \rightarrow \overline{\mathrm{R}}$ is said to be quasiconvex when its (lower) level sets $S_{t}(f)=\{x: f(x) \leq t\}$ are convex. A convex function $f$ is necessarily quasiconvex, the composition $g=k \circ f$ of a quasiconvex function $f$ with a scaling (increasing and continuous) function $k$ is quasiconvex. A maximum of quasiconvex functions is quasiconvex but their sum is not, the ratio of a positive convex function over a positive concave function is quasiconvex but not convex.

A basic ingredient of the theory of the consumer behavior is the concept of a preference relation. When faced with prices of goods and services, the consumer makes his choice according to his preferences and his budget constraint.

Jean-Pierre Crouzeix

LIMOS, Université Clermont Auvergne

1 rue de la Chebarde, 63178 Aubière, France

jp.crouzeix@isima.fr 
Gérard Debreu [10] has shown that, under rational assumptions, the choice is determined in maximizing a continuous increasing quasiconcave function, called utility, under the budget constraint (a function $u$ is said to be quasiconcave if $-u$ is quasiconvex). This utility function $u$ is unique up to a scaling function $k$ : if $u$ is a utility function describing the behavior of the consumer, so is $k \circ u$. One question: given a quasiconcave utility function, does there exist some scaling function $k$ which makes $k \circ u$ concave? If yes $u$ is said to be concavifiable. But, in general, there are no reasons for a quasiconcave utility function to be concavifiable.

G. Debreu [11] has shown that, when the preference relation admits a representation by a concave utility function, there exists a representation $\bar{u}$ concave such that for each other concave representation $u$ there exists a concave scaling function $k$ with $u=k \circ \bar{u}$. This utility function $\bar{u}$ is said to be a least concave representation of the preference relation, it is unique up to a scaling function $k$ of type $k(t)=\alpha t+\beta, \alpha>0$. Let us show the interest of a least concave utility representation.

Let us consider an investment $c$ (for instance in a portfolio selection). The value of this investment after the fixed period is given by $u\left(Z_{c}\right)$ where $Z_{c}$ is a random value and $u$ is a utility function. Let $v=k \circ u$ with $k$ increasing and concave. It is convenient to compare the expected value $E\left(v\left(Z_{c}\right)\right)$ of $v\left(Z_{c}\right)$ with $k\left(E\left(u\left(Z_{c}\right)\right)\right)$ in order to work on comparable quantities. The Jensen inequality [17] says that

$$
E\left(u\left(Z_{c}\right)\right) \geq k^{-1}\left[E\left(v\left(Z_{c}\right)\right)\right] .
$$


The expected value is greater with a least concave utility function.

Let us now discuss insurances against risks. Let $x \in \mathrm{R}^{\mathrm{n}}$ (estate, goods, services, money, ...) faced with the risk expressed by the random vector $Z$ of $\mathrm{R}^{\mathrm{n}}$, the value of $x$ is expressed by $u(x)$ where $u$ is a utility function associated to the personal preferences. Two possibilities: to accept the risk and the value of $x$ becomes $u(x-Z)$ or to insure $x$ against the risk and then to pay the amount of insurance $\lambda a$, the value of $x$ becomes then $u(x-\lambda a)$. A fair way to fix $\lambda a$ is through the equation $E(u(x-Z))=u(x-\lambda a)$. Another representation of the preferences $v=k \circ u$ would get another value $\mu a$ for the amount of insurance. If $k$ is concave, the Jensen inequality gives $\lambda \leq \mu$. Using $v$ instead of $u$ corresponds to a greater aversion against risks. This example shows again the reasons for interest of least concave utilities.

In this paper, we give characterizations for a quasiconvex function to be convex. Given a quasiconvex function $f$, we study what conditions on $k$ are necessary to get $k \circ f$ convex, least convex. Finally, we show that, for a function as smooth as the function $f$ defined on the positive orthant of $\mathrm{R}^{2}$ by $f\left(x_{1}, x_{2}\right)=x_{2} / x_{1}$, there is no scaling function $k$, no $\bar{x}>0$ and no neighborhood $V$ of $\bar{x}$ on which $k \circ f$ is convex. Hence, quasiconvex fractional programs cannot be transformed in convex programs because they involve ratios of functions. We close the paper with approximations of a preorder from a finite number of observations and we give a few words on the very hard revealed preference problem. 
2 Notation.

A subset $C$ of $\mathrm{R}^{\mathrm{n}}$ is said to be convex if $x+t(x-y) \in C$ when $x, y \in C$ and $t \in[0,1]$. The relative interior of $C$, denoted by ri $(C)$, is the interior of $C$ when considered as a subset of its affine subspace.

Assume that $C$ is a nonempty closed convex set and $x \in C$ is fixed, the set

$$
N_{C}(x):=\left\{x^{*} \in \mathrm{R}^{\mathrm{n}}:\left\langle\mathrm{x}^{*}, \mathrm{y}-\mathrm{x}\right\rangle \leq 0 \quad \forall \mathrm{y} \in \mathrm{C}\right\},
$$

called the normal cone at $x$ is a closed convex cone. $0 \in N_{C}(x), N_{C}(x)=\{0\}$ if and only if $x \in \operatorname{int}(C)$.

Let $f: \mathrm{R}^{\mathrm{n}} \rightarrow[-\infty,+\infty]$ be a given function. The epigraph of $f$ is the set $\operatorname{epi}(f)=\left\{(x, t) \in \mathrm{R}^{\mathrm{n}} \times \mathrm{R}: \mathrm{f}(\mathrm{x}) \leq \mathrm{t}\right\}$, its lower level subsets are the sets $S_{t}(f)=\left\{x \in \mathrm{R}^{\mathrm{n}}: \mathrm{f}(\mathrm{x}) \leq \mathrm{t}\right\}$ and the upper level sets are the sets $S_{t}^{+}(f)=$ $\left\{x \in \mathrm{R}^{\mathrm{n}}: \mathrm{f}(\mathrm{x}) \geq \mathrm{t}\right\}$. The domain of $f$ is the set $\operatorname{dom}(f)=\{x: f(x)<+\infty\}$.

One has

$$
f(x)=\min _{t}[t:(x, t) \in \operatorname{epi}(f)]=\min _{t}\left[t: x \in S_{t}(f)\right] .
$$

It is common to omit the qualification lower when working on lower level subsets.

A function $f$ is said to be lower semi-continuous (lsc) at $x$ if for each $\lambda<f(x)$ a neighborhood $V$ of $x$ exists such that $f(y)>\lambda$ for all $y \in V$. It is known that $f$ is lsc on $\mathrm{R}^{\mathrm{n}}$ if and only if its epigraph is closed, or equivalently if and only if all its level sets are closed.

A function $f$ is said to be positively homogeneous if $f(0)=0$ and $f(\lambda x)=\lambda f(x)$ for all $x \in \mathrm{R}^{\mathrm{n}}$ and all $\lambda>0$. 
A function $f$ is said to be convex if its epigraph is convex, quasiconvex if its lower level sets are convex, quasiconcave if its upper level sets are convex. It is clear that a convex function is quasiconvex and that $f$ is quasiconcave if and only if $-f$ is quasiconvex.

The indicator function of $C \subseteq \mathrm{R}^{\mathrm{n}}$ is the function $\delta_{C}$ defined by $\delta_{C}(x)=0$ if $x \in C$ and $\delta_{C}(x)=+\infty$ if not. A set $C$ is closed and convex if and only if its indicator function is convex and lower semi-continuous.

A direct study of the epigraph shows that if a convex function $f$ takes the value $-\infty$ at some point then $f(x)=-\infty$ if $x \in \operatorname{ri}(\operatorname{dom}(f))$ and $f(x)=+\infty$ if $x \notin \operatorname{dom}(f)$. If, in addition $f$ is lsc, then it takes only the values $-\infty$ and $+\infty$. With regard to these observations, a function is said to be proper if it does not take the value $-\infty$, its domain is not empty.

\section{Geometry and Duality.}

Next, we present a brief, very geometric and unconventional, overview on the duality on closed convex sets, proper lsc convex functions functions and lsc quasiconvex functions.

a) With $C \subseteq \mathrm{R}^{\mathrm{n}}$ closed nonempty convex, we associate its support function $\delta_{C}^{*}$ defined by

$$
\delta_{C}^{*}\left(x^{*}\right):=\sup _{y \in C}\left\langle x^{*}, y\right\rangle=\sup _{y}\left[\left\langle x^{*}, y\right\rangle-\delta_{C}(y)\right]
$$

This function is convex and lsc as a supremum of lsc convex functions, it is also positively homogeneous. 
If $x \notin C$, there exist $x^{*}$ and $\alpha$ such that $\left\langle x^{*}, x\right\rangle>\alpha>\left\langle x^{*}, y\right\rangle$ for all $y \in C$. If $x \in C,\left\langle x^{*}, x\right\rangle \leq \delta_{C}^{*}\left(x^{*}\right)$ and when the equality holds $\left\langle x^{*}, y-x\right\rangle \leq 0$ for all $y \in C$ and therefore $x^{*} \in N_{C}(x)$. In conclusion,

$$
\delta_{C}(x)=0 \Longleftrightarrow x \in C \Longleftrightarrow\left\langle x^{*}, x\right\rangle-\delta_{C}^{*}\left(x^{*}\right) \leq 0 \quad \forall x^{*}
$$

And finally,

$$
\delta_{C}(x)=\sup _{y^{*}}\left[\left\langle y^{*}, x\right\rangle-\delta_{C}^{*}\left(y^{*}\right)\right]
$$

to be compared with (1).

The domain of $\delta_{C}^{*}$, called the barrier cone of $C$, is denoted by $\operatorname{barr}(C)$.

By definition,

$$
\operatorname{barr}(C)=\left\{x^{*}: \delta_{C}^{*}\left(x^{*}\right)<+\infty\right\}
$$

It is easily seen that a closed convex set is compact if and only if its barrier cone is the whole space.

b) Let $f$ be a proper lsc convex function on $\mathrm{R}^{\mathrm{n}}$. Set $C=\operatorname{epi}(f)$.

$$
\delta_{C}^{*}\left(x^{*}, \lambda^{*}\right)=\sup _{x, \lambda}\left[\left\langle x^{*}, x\right\rangle+\lambda^{*} \lambda: f(x) \leq \lambda\right]
$$

Clearly

$$
\delta_{C}^{*}\left(x^{*}, \lambda^{*}\right)= \begin{cases}+\infty & \text { if } \lambda^{*}>0 \\ \delta_{\operatorname{dom}(f)}^{*}\left(x^{*}\right) & \text { if } \lambda^{*}=0 \\ \left|\lambda^{*}\right| \delta_{C}^{*}\left(\frac{x^{*}}{\left|\lambda^{*}\right|},-1\right) & \text { if } \lambda^{*}<0\end{cases}
$$

In view of $(2)$

$$
(x, \lambda) \in \operatorname{epi}(f) \Longleftrightarrow \sup _{x^{*}, \lambda^{*}}\left[\left\langle x^{*}, x\right\rangle+\lambda^{*} \lambda-\delta_{C}^{*}\left(x^{*}, \lambda^{*}\right)\right] \leq 0 .
$$


The inequality holds for $\lambda^{*}>0$. For $\lambda^{*}=0$, one obtains $x \in \operatorname{dom}(f)$ and for $\lambda^{*}<0$ one obtains

$$
\left|\lambda^{*}\right|\left[\left\langle\frac{x^{*}}{\left|\lambda^{*}\right|}, x\right\rangle-\lambda-\delta_{C}^{*}\left(\frac{x^{*}}{\left|\lambda^{*}\right|},-1\right)\right] \leq 0 \quad \forall x^{*}, \quad \forall \lambda^{*}<0 .
$$

Thus, $(x, \lambda) \in \operatorname{epi}(f)$ if and only if $\sup _{y^{*}}\left[\left\langle y^{*}, x\right\rangle-\delta_{C}^{*}\left(y^{*},-1\right)\right] \leq \lambda$.

The function $f^{*}$ defined by $f^{*}\left(x^{*}\right)=\delta_{C}^{*}\left(x^{*},-1\right)$ is lsc, proper and convex. It called the conjugate function of $f$. The duality between $f$ and $f^{*}$ is quite symmetric since

$$
f^{*}\left(x^{*}\right)=\sup _{x}\left[\left\langle x^{*}, x\right\rangle-f(x)\right] \forall x^{*} \text { and } f(x)=\sup _{x^{*}}\left[\left\langle x^{*}, x\right\rangle-f^{*}\left(x^{*}\right)\right] \forall x \text {. }
$$

c) Next, let $f$ be a lsc quasiconvex function on $\mathrm{R}^{\mathrm{n}}$. By definition, its level sets $S_{t}(f)=\{x: f(x) \leq t\}, t \in \mathrm{R}$ are closed and convex. Let us associate with $f$ the function $F$ defined on $\mathrm{R}^{\mathrm{n}} \times \mathrm{R}$ by

$$
F\left(x^{*}, t\right)=\delta_{S_{t}(f)}^{*}\left(x^{*}\right)=\sup _{x}\left[\left\langle x^{*}, x\right\rangle: f(x) \leq t\right] .
$$

The function $F$ is lsc, convex and positively homogeneous in the first variable, nondecreasing in the second one. In view of the equivalence (2)

$$
f(x) \leq t \Longleftrightarrow x \in S_{t}(f) \Longleftrightarrow F\left(x^{*}, t\right) \geq\left\langle x^{*}, x\right\rangle \quad \forall x^{*},
$$

from which,

$$
f(x)=\inf _{t}\left[t: F\left(x^{*}, t\right)-\left\langle x^{*}, x\right\rangle \geq 0 \forall x^{*}\right]
$$

With regard to (3) and (4), we consider $F$ as $f$ as dual functions of each other. 


\section{When a Quasiconvex Function Dreams To Be Convex.}

The following theorem characterizes convex functions among quasiconvex functions.

Theorem 4.1 [3,4] Let $f$ be a lsc quasiconvex function. The function $f$ is convex if and only for each $x^{*}$ the function $F_{x^{*}}: t \rightarrow F\left(x^{*}, t\right)$ is concave.

Proof a) Assume $f$ convex. Let $x^{*}$ be fixed, the function $\theta_{x^{*}}$ defined by

$$
\theta_{x^{*}}(x, t)=\left\langle x^{*}, x\right\rangle-\delta_{\mathrm{epi}(f)}(x, t) .
$$

is concave in $(x, t)$. Hence, the function $t \rightarrow F\left(x^{*}, t\right)=\sup _{x} \theta_{x^{*}}(x, t)$ is concave.

b) Conversely, assume the concavity of functions $F_{x^{*}}$. Let us define the set $A$ and the function $\varphi$ by

$$
A=\left\{(x, t): F\left(x^{*}, t\right)-\left\langle x^{*}, x\right\rangle \geq 0 \forall x^{*}\right\}, \quad \varphi(x, t)=t+\delta_{A}(x, t) .
$$

The set $A$ is convex and the function $\varphi$ is convex. Next, the function $f$ is convex since $f(x)=\inf _{t} \varphi(x, t)$ for all $x$.

Let us place in the case where $f$ is a proper lsc convex function with $m=\inf f(x)$. The following results are direct consequences of the concavity of the functions $F\left(x^{*},.\right)$

1. $F\left(x^{*}, t\right) \leq F\left(x^{*}, t^{\prime}\right) \quad \forall t<t^{\prime}$.

2. If $F\left(x^{*}, t\right)=+\infty$ for some $t>m$ then $F\left(x^{*}, t^{\prime}\right)=+\infty$ for all $t^{\prime}>m$.

3. If $F\left(x^{*}, t\right)=F\left(x^{*}, t^{\prime}\right)$ for some $t^{\prime}>t \geq m$, then $F\left(x^{*}, t\right)=F\left(x^{*}, t^{\prime \prime}\right)$ for all $t^{\prime \prime}>t$. 
4. $\left[t^{\prime \prime}-t^{\prime}\right]\left[F\left(x^{*}, t^{\prime}\right)-F\left(x^{*}, t\right)\right] \geq\left[t^{\prime}-t\right]\left[F\left(x^{*}, t^{\prime \prime}\right)-F\left(x^{*}, t^{\prime}\right)\right]$

for all $t^{\prime \prime}>t^{\prime}>t \geq m$.

An improvement of the second statement, due to Fenchel, says that the nonempty lower level sets of a proper lsc convex function have the same barrier cones.

\section{Convex Interpolation.}

$C_{0}, C_{1}, \cdots, C_{p}$ being $p+1$ nonempty closed convex subsets of $R^{n}$ such that $C_{i} \subseteq \operatorname{int}\left(C_{i+1}\right)$ for $i=0, \cdots, p-1$, our present challenge consists in finding $p+1$ numbers $t_{0}<t_{1}<\cdots<t_{p}$ and a convex function $f$ with $C_{i}=\left\{x: f(x) \leq t_{i}\right\}$ for all $i$. The following requirements on the sets $C_{i}$ are needed:

1. The sets $C_{i}$ share the same barrier cones. Denote by $K$ this cone.

2. If $\delta_{C_{i}}^{*}\left(x^{*}\right)=\delta_{C_{i+1}}^{*}\left(x^{*}\right)$, then $\delta_{C_{i}}^{*}\left(x^{*}\right)=\delta_{C_{j}}^{*}\left(x^{*}\right)$ for all $j>i+1$.

3. For $i=0,1, \cdots, p-2$ and $x^{*} \in K$,

$$
\left[t_{i+2}-t_{i+1}\right]\left[\delta_{C_{i+1}}^{*}\left(x^{*}\right)-\delta_{C_{i}}^{*}\left(x^{*}\right)\right] \geq\left[t_{i+1}-t_{i}\right]\left[\delta_{C_{i+2}}^{*}\left(x^{*}\right)-\delta_{C_{i+1}}^{*}\left(x^{*}\right)\right]
$$

We are ready to describe the interpolation process.

1. Initialisation: Take $t_{0}, t_{1}$ be such that $t_{0}<t_{1}$. For $i=0,1, \cdots, p-2$

2. Building $t_{i}, t_{2}, \cdots, t_{p}$ : In succession for $i=0,1, \cdots, p-2$ and in concordance with requirements 2 and 3 we compute

$$
\lambda_{i}=\sup _{x^{*}}\left[\frac{\delta_{C_{i+2}}^{*}\left(x^{*}\right)-\delta_{C_{i+1}}^{*}\left(x^{*}\right)}{\delta_{C_{i+1}}^{*}\left(x^{*}\right)-\delta_{C_{i}}^{*}\left(x^{*}\right)}: 0 \neq x^{*} \in K, \delta_{C_{i}}^{*}\left(x^{*}\right) \neq \delta_{C_{i+1}}^{*}\left(x^{*}\right)\right] .
$$

If $\lambda_{i}=+\infty$, STOP: There is no solution to the problem.

If $\lambda_{i}<+\infty$, take $t_{i+2}=t_{i+1}+\lambda_{i}\left(t_{i+1}-t_{i}\right)$.

By construction, $0<\lambda_{i}<+\infty$ for all $i$ and $t_{0}<t_{1}<\cdots<t_{p}$. 
3. Construction of $F\left(x^{*},.\right)$ :

(a) If $t<t_{0}: F\left(x^{*}, t\right)=-\infty$

(b) If $x^{*} \notin K$ and $t \geq t_{0}: F\left(x^{*}, t\right)=+\infty$.

(c) If $x^{*} \in K$ and $t_{i} \leq t \leq t_{i+1}, i=0, \cdots, p-1$ :

$$
F\left(x^{*}, t\right)=\frac{t_{i+1}-t}{t_{i+1}-t_{i}} \delta_{C_{i}}^{*}\left(x^{*}\right)+\frac{t-t_{i}}{t_{i+1}-t_{i}} \delta_{C_{i+1}}^{*}\left(x^{*}\right) .
$$

(d) If $t_{p}<t: F\left(x^{*}, t\right)=F\left(x^{*}, t_{p}\right)$.

For each $t \in\left[t_{0}, t_{p}\right], F(., t)$ is the support function of a closed convex set, this set is $C_{i}$ when $t=t_{i}$. For each vector $x^{*} \in K$, the function $F\left(x^{*},.\right)$ is piecewise linear and continuous on $\left[t_{0}, t_{p}\right]$, besides $F\left(x^{*}, t_{i}\right)=\delta_{C_{i}}^{*}\left(x^{*}\right)$ for each $i=0, \cdots, p$.

By construction, for any $i=0, \cdots, p-2$ and for any $x^{*} \in K$,

$$
\frac{t_{i+2}-t_{i+1}}{t_{i+1}-t_{i}}=\lambda_{i} \geq \frac{\delta_{C_{i+2}}^{*}\left(x^{*}\right)-\delta_{C_{i+1}}^{*}\left(x^{*}\right)}{\delta_{C_{i+1}}^{*}\left(x^{*}\right)-\delta_{C_{i}}^{*}\left(x^{*}\right)}=\frac{F\left(x^{*}, t_{i+2}\right)-F\left(x^{*}, t_{i+1}\right)}{F\left(x^{*}, t_{i+1}\right)-F\left(x^{*}, t_{i}\right)},
$$

and therefore

$$
\frac{F\left(x^{*}, t_{i+2}\right)-F\left(x^{*}, t_{i+1}\right)}{t_{i+2}-t_{i+1}} \leq \frac{F\left(x^{*}, t_{i+1}\right)-F\left(x^{*}, t_{i}\right)}{t_{i+1}-t_{i}} .
$$

The concavity of the functions $F\left(x^{*},.\right)$ is a consequence of the piecewise linearity of these functions and the inequalities on the left and right derivatives at points $t_{i+1}$. The function $f$ obtained from $F$ through relation (4) is convex and responds to the question.

Let us consider the special case where the sets $C_{i}$ are bounded.

Corollary 5.1 [20,21] Let $C_{0}, C_{1}, \cdots, C_{p}$ be $p+1$ nonempty closed bounded convex sets such that $C_{i} \subseteq \operatorname{int}\left(C_{i+1}\right)$ for $i=0, \cdots, p-1$. Then, there exists $f$ convex and lsc and $t_{0}<t_{1}<\cdots<t_{p}$ such that $C_{i}=\left\{x: f(x) \leq t_{i}\right\}$ for all $i$. 
Proof : Under the assumptions the barrier cone $K$ is the whole space, the quantities $\delta_{C_{i+1}}^{*}\left(x^{*}\right)-\delta_{C_{i}}^{*}\left(x^{*}\right)$ are continuous and positive, the quantities $\lambda_{i}$ are finite, the function $F$ therefore is well defined.

Let us point out a recent and very interesting paper of Bolte and Pauwels [2] dealing with related questions.

\section{To Be Convexifiable?}

\subsection{The General Case}

Assume that $g=k \circ f$ is convex. The three following conditions are direct translations from $g$ to $f$ of the analogous conditions due to the convexity of $g$, they are necessary for the convexifiability of $f$ :

1) If $\inf f(x)<\lambda<\mu$, the level sets $S_{\lambda}(f)$ and $S_{\mu}(f)$ have the same dimension.

2) The barrier cones of the nonempty level sets of $f$ are the same.

3) If $\inf f(x)<\lambda<\mu$ and $F_{x^{*}}(\lambda)=F_{x^{*}}(\mu)$, then $F_{x^{*}}(\lambda)=F_{x^{*}}(t)$ for all $t>\lambda$.

The last property is due to the fact that the function $G_{x^{*}}$ is concave and nondecreasing. The quasiconvex functions $f$ and $g$ defined on the positive orthant of $\mathrm{R}^{2}$ by $f\left(x_{1}, x_{2}\right)=-g\left(x_{1}, x_{2}\right)=x_{2} / x_{1}$ are not convexifiable because of the second condition.

Finding $k$ for which $k \circ f$ is convex or least convex is a very hard problem. Indeed, for each $t$, the convexity of all functions $g_{x, d}: s \rightarrow k(f(x+s d))$ in a 
same neighborhood of 0 is required for all $d \in \mathrm{R}^{\mathrm{n}}$ and all $x$ such that $f(x)=t$.

One way is to concentrate in one function the whole information corresponding at all points $x$ such that $f(x)=t$, this is precisely what the dual function

\section{$F$ does to be associated with $f$.}

If $g=k \circ f$ with $k$ increasing and continuous, the relationships between the dual functions $F$ and $G$ associated with $f$ and $g$ and $f$ are as follows

$$
\begin{gathered}
G\left(x^{*}, s\right)=\sup _{x}\left[\left\langle x^{*}, x\right\rangle: k(f(x)) \leq s,\right]=F\left(x^{*}, k^{-1}(s)\right)=F_{x^{*}} \circ k^{-1}(s) \\
F\left(x^{*}, t\right)=\sup _{x}\left[\left\langle x^{*}, x\right\rangle: k^{-1}(g(x)) \leq t\right]=G\left(x^{*}, k(t)\right) .
\end{gathered}
$$

Hence, the problem consists in finding $k$ increasing and continuous which make all functions $F_{x^{*}} \circ k^{-1}$ concave. Because $F$ is positively homogeneous in $x^{*}$, it is enough to consider the vectors $x^{*}$ having norm 1 .

Set $I=f\left(\mathrm{R}^{\mathrm{n}}\right), F_{x^{*}} \circ k^{-1}$ is concave if and only if, for all $s_{-}, s, s_{+} \in f(I)$ such that $s_{-}<s<s_{+}$

$$
\frac{F_{x^{*}} \circ k^{-1}(s)-F_{x^{*}} \circ k^{-1}\left(s_{-}\right)}{s-s_{-}} \geq \frac{F_{x^{*}} \circ k^{-1}\left(s_{+}\right)-F_{x^{*}} \circ k^{-1}(s)}{s_{+}-s}
$$

or, equivalently, if and only if, for all $t_{-}, t, t_{+} \in I$ such that $t_{-}<t<t_{+}$

$$
\frac{F_{x^{*}}(t)-F_{x^{*}}\left(t_{-}\right)}{k(t)-k\left(t_{-}\right)} \geq \frac{F_{x^{*}}\left(t_{+}\right)-F_{x^{*}}(t)}{k\left(t_{+}\right)-k(t)} .
$$

Recall that $F_{x^{*}}\left(t_{+}\right)-F_{x^{*}}(t)=0$ as soon as $F_{x^{*}}(t)-F_{x^{*}}\left(t_{-}\right)=0$. The function $g=k \circ f$ is convex if and only if for any $t_{-}, t, t_{+} \in I$ such that $t_{-}<t<t_{+}$

$$
\sup _{x^{*} \neq 0}\left[\frac{F_{x^{*}}\left(t_{+}\right)-F_{x^{*}}(t)}{F_{x^{*}}(t)-F_{x^{*}}\left(t_{-}\right)}: F_{x^{*}}(t)>F_{x^{*}}\left(t_{-}\right)\right] \leq \frac{k\left(t_{+}\right)-k(t)}{k(t)-k\left(t_{-}\right)}
$$


It follows that a convex function $f$ is least convex if and only if the only concave functions $k$ for which condition (6) holds are of the type $k(t)=\alpha t+\beta$ with $\alpha>0$.

Let us prove that the function $f$ built in section 5 is least convex. For all $t_{-}, t, t_{+} \in\left[t_{i}, t_{i+1}\right]$ such that $t_{-}<t<t_{+}$condition (6) becomes

$$
\frac{t_{+}-t}{t-t_{-}} \leq \frac{k\left(t_{+}\right)-k(t)}{k(t)-k\left(t_{-}\right)}
$$

Hence, there exist $\alpha_{i}, \beta_{i}$ such that $k(t)=\alpha_{i} t+\beta_{i}$ for all $t \in\left[t_{i}, t_{i+1}\right]$. Next, from condition (6) again and the construction of the sequence $\left\{t_{i}\right\}$

$$
\frac{F_{x^{*}}\left(t_{i+2}\right)-F_{x^{*}}\left(t_{i+1}\right)}{F_{x^{*}}\left(t_{i+1}\right)-F_{x^{*}}\left(t_{-} i\right)} \leq \frac{k_{+}^{\prime}\left(t_{i+1}\right)\left(t_{i+2}-t_{i+1}\right)}{k_{-}^{\prime}\left(t_{i+1}\right)\left(t_{i+1}-t_{i}\right)}=\lambda_{i} \frac{\alpha_{i+1}}{\alpha_{i}} .
$$

Then, relation (5) implies $\alpha_{i+1} \geq \alpha_{i}$ and, because the function $k$ is concave, $\alpha_{i+1}=\alpha_{i}$. Finally, the continuity of the function $k$ implies $\beta_{i+1}=\beta_{i}$. We have proved that $k$ concave with $k \circ f$ convex is possible only if $k$ is of the type $k(t)=\alpha t+\beta$.

\section{Quasiconvex Positively Homogeneous Functions.}

Let $f: \mathrm{R}^{\mathrm{n}} \rightarrow(-\infty,+\infty]$ be a lsc quasiconvex positively homogeneous function with $n$

1. $F_{x^{*}}(t)=-t F_{x^{*}}(-1)$ if $t<0$

2. $F_{x^{*}}(t)=t F_{x^{*}}(1)$ if $t>0$,

3. $F_{x^{*}}(-1) \leq F_{x^{*}}(0) \leq F_{x^{*}}(1)$.

We consider the three following cases 
a) $K=\emptyset$. Then $F_{x^{*}}$ is concave for all $x^{*}$ and therefore $f$ is convex. It is also least convex. Being positively homogeneous, $f$ is the support function of a closed convex set $A$. Assume, for contradiction, $0 \notin A$, let $p$ be the projection of 0 on $A$. Then

$$
0<\|p\|^{2} \leq \inf _{x^{*}}\left[\left\langle p, x^{*}\right\rangle: x^{*} \in A\right]=-f(-p),
$$

in contradiction with $f(x) \geq 0$ for all $x$. Thus $0 \in A$ and $\lambda A \subseteq A$ whenever $0<\lambda<1$

b) $\operatorname{dom}(f)=\bar{K}$. Then, $F_{x^{*}}(t)=F_{x^{*}}(0)$ for all $t>0$. Next, $F_{x^{*}}$ is concave for all $x^{*}$ and therefore $f$ is convex. Moreover, $f$ is least convex and it is the support function of a closed convex set $B$. One has $0 \notin B$ because $K \neq \emptyset$. Since $f \leq \delta_{\bar{K}}$ one has $\delta_{B}=f^{*} \geq \delta_{\bar{K}}^{*}=\delta_{K^{\circ}}$ and therefore $B \subseteq K^{\circ}$. Finally, one easily shows that $\lambda B \subseteq B$ when $\lambda>1$.

3) It remains to treat the case where $f$ takes both positive and negative values. Let us introduce the functions $f_{-}$and $f_{+}$as follows

$$
f_{-}(x)=\left\{\begin{array}{ll}
f(x) \text { if } x \in \bar{K}, \\
+\infty \text { otherwise. }
\end{array} \quad f_{+}(x)=\left\{\begin{array}{cc}
0 & \text { if } x \in \bar{K} \\
f(x) & \text { otherwise }
\end{array}\right.\right.
$$

By construction, $f=\min \left[f_{-}, f_{+}\right]$. The two functions $f_{-}$and $f_{+}$are lsc, convex and positively homogeneous, they are the support functions of two closed convex sets $C_{-}$and $C_{+}$.

In conclusion, a proper, lsc, quasiconvex and positively homogeneous functions is the minimum of two proper, lsc, convex, positively homogeneous functions. 
From the inequalities

$$
\delta_{C_{-}}^{*}=f_{-} \leq \delta_{\bar{K}}=\delta_{K^{\circ}}^{*} \quad \text { and } \quad \delta_{C_{+}}^{*}=f_{+} \leq \delta_{\bar{K}}=\delta_{K^{\circ}}^{*}
$$

one obtains

$$
\delta_{C_{-}}=f_{-}^{*} \geq \delta_{\frac{k}{K}}^{*}=\delta_{K^{\circ}} \quad \text { and } \quad \delta_{C_{+}}=f_{+}^{*} \geq \delta_{\bar{K}}^{*}=\delta_{K^{\circ}}
$$

and finally

$$
\lambda C_{-} \subseteq C_{-} \subseteq K^{\circ} \forall \lambda \geq 1 \quad \text { and } \quad \mu C_{+} \subseteq C_{+} \subseteq K^{\circ} \forall \mu \in[0,1]
$$

As an application, let us consider the case where $g$ is a quasiconvex function continuous at a point $a$ with finite directional derivatives $g^{\prime}(a, d)$ at $a$ along all directions $d$. Then, $g^{\prime}(a,$.$) is quasiconvex [5,6]$ and therefore is the minimum of the support functions of two closed convex sets $\partial_{-} g(a)$ and $\partial_{+} g(a)$. The result holds also for upper Dini-directional derivatives but not for the lower ones.

\section{Back to the Ratio $x_{2} / x_{1}$.}

We have already seen that the function $f$ defined by $f\left(x_{1}, x_{2}\right)=x_{2} / x_{1}$ is not convexifiable on the whole positive orthant. Is it locally convexifiable?

Assume that there exists $k$ increasing and twice differentiable such that $g=k[\ln (f)]$ is convex in a neighborhood of a point $a$ in the positive orthant. Then, in this neighborhood, $\operatorname{det}\left(\nabla^{2} g(x)\right)=-\left[k^{\prime}\left(\ln (f(x)) / x_{1} x_{2}\right]^{2}<0\right.$, in contradiction with the convexity (and also the concavity) of $g$. Hence $f$ is not 
convexifiable and not concavifiable via a twice differentiable scaling function.

What about a non differentiable scaling function $k$ ?

Let us place at the point $e=(1,1)$ and let us consider the neighborhoods of type $B_{r}=\left\{x \in \mathrm{R}^{2}:\left(\mathrm{x}_{1}-1\right)^{2}+\left(\mathrm{x}_{2}-1\right)^{2} \leq \mathrm{r}^{2}\right\}$ with $\left.r \in\right] 0,1[$ chosen as small as wanted.

1) $x^{*} \in \mathrm{R}^{2}$ being fixed, let us compute the function

$$
F_{x^{*}}(t)=\sup _{x}\left[\left\langle x^{*}, x\right\rangle: x \in B_{r}(t)\right] \quad \text { where } \quad B_{r}(t)=\left\{x \in B_{r}: f(x) \leq t\right] .
$$

The function $F_{x^{*}}$ is nondecreasing. The supremum is reached in an extremal point of $B_{r}(t)$ when this set is non empty. Let $x=\left(x_{1}, x_{2}\right)$ be such that $f(x)=t$ and $\|x-e\|=r$, then $x_{1}$ and $x_{2}$ are solutions of the equation

$$
\left(1+t^{2}\right) x_{1}^{2}-2(1+t) x_{1}+\left(2-r^{2}\right)=0, \quad x_{2}=t x_{1} .
$$

Such $x_{1}$ exist only when $\left(r^{2}-1\right) t^{2}+2 t+r^{2}-1 \geq 0$. Set

$$
t_{-}=\frac{1}{1-r^{2}}\left(1-r \sqrt{2-r^{2}}\right), \quad t_{+}=\frac{1}{1-r^{2}}\left(1+r \sqrt{2-r^{2}}\right) .
$$

If $t<t_{-}$then $B_{r}(t)=\emptyset$ and $F_{x^{*}}(t)=-\infty$.

If $t>t_{+}$then $B_{r}(t)=B_{r}$ and $\left\langle x^{*}, x\right\rangle$ reaches its maximum on $B_{r}$ at point $a=e+\left\|x^{*}\right\|^{-1} x^{*}$. Then, $F_{x^{*}}(t)=\left\langle e, x^{*}\right\rangle+r\left\|x^{*}\right\|$.

It remains to consider $t_{-} \leq t \leq t_{+}$. Let again $a=e+\left\|x^{*}\right\|^{-1} x^{*}$.

In case where $a_{2} \leq t a_{1},\left\langle x^{*}, x\right\rangle$ reaches its maximum on $B_{r}(t)$ at $a$. Hence $F_{x^{*}}(t)=\left\langle e, x^{*}\right\rangle+r\left\|x^{*}\right\|$. Let us note that the inequality $a_{2} \leq t a_{1}$ is equivalent to the inequality $t \geq\left(\left\|x^{*}\right\|+x_{1}^{*}\right)^{-1}\left(\left\|x^{*}\right\|+x_{2}^{*}\right)$. 
Next, assume $t \leq\left(\left\|x^{*}\right\|+x_{1}^{*}\right)^{-1}\left(\left\|x^{*}\right\|+x_{2}^{*}\right)$. The maximum of $\left\langle x^{*}, x\right\rangle$ on $B_{r}(t)$ is reached at one of the two points $b(t)$ and $c(t)$ where the straight line $x_{2}-t x_{1}=0$ intersects the boundary of $B_{r}$. An easy computation gives

$$
\begin{array}{ll}
b_{1}(t)=\frac{1}{1+t^{2}}\left[1+t+\sqrt{\left(1-r^{2}\right)\left(t-t_{-}\right)\left(t_{+}-t\right)}\right], & b_{2}(t)=t b_{1}(t), \\
c_{1}(t)=\frac{1}{1+t^{2}}\left[1+t-\sqrt{\left(1-r^{2}\right)\left(t-t_{-}\right)\left(t_{+}-t\right)}\right], & c_{2}(t)=t c_{1}(t) .
\end{array}
$$

From what one deduces

1. If $x_{1}^{*}+t x_{2}^{*} \geq 0$,

$$
F_{x^{*}}(t)=\frac{x_{1}^{*}+t x_{2}^{*}}{1+t^{2}}\left[1+t+\sqrt{\left(1-r^{2}\right)\left(t-t_{-}\right)\left(t_{+}-t\right)}\right]
$$

2. If $x_{1}^{*}+t x_{2}^{*} \leq 0$,

$$
F_{x^{*}}(t)=\frac{x_{1}^{*}+t x_{2}^{*}}{1+t^{2}}\left[1+t-\sqrt{\left(1-r^{2}\right)\left(t-t_{-}\right)\left(t_{+}-t\right)}\right]
$$

The right and the left derivatives of $F_{x^{*}}$ coincide at any $t$ except when $x_{1}^{*}+t x_{2}^{*}=0$ where the right one is strictly greater than the left one.

2) Assume, for contradiction, the existence of $k$ increasing and continuous for which $g=k \circ f$ is convex on $B_{r}$. The convexity of $g$ implies the concavity of $G_{x^{*}} . F_{x^{*}}$ and $G_{x^{*}}$ are connected by the relation

$$
G_{x^{*}}(s)=\sup _{x}\left[\left\langle x^{*}, x\right\rangle:\|x-e\| \leq r, k(f(x)) \leq s\right]=F_{x^{*}}\left(k^{-1}(s)\right) .
$$

On the other hand, since $k(s)=k(f(1, s))=g(1, s)$ for all $s$, the function $k$ is convex, $k^{-1}$ is concave and therefore almost everywhere differentiable. Let us consider a point $t$ where $k^{-1}$ is differentiable, next choose $x^{*}$ such that $x_{1}^{*}+t x_{2}^{*}=0$ with $x_{2}^{*}>0$. Then the right derivative of $G_{x^{*}}$ at $t$ is strictly greater than the left derivative, in contradiction with the concavity of $G_{x^{*}}$. 
We have just shown that $f$ is not convexifiable on $B_{r}$. Because $r>0$ can be chosen as small as wanted, $f$ is not locally convexifiable at $e$. This result is extended to any point in the positive orthant via a linear transformation. A similar proof shows that $f$ is also not locally concavifiable on the positive orthant. In conclusion, "Quand on ne peut pas, on ne peut pas" [23].

\section{On the Approximation of Preferences by Utility Functions.}

9.1 Statement of the problem

Let us return to the situation where the consumption of a consumer is submitted to the preorder $\preceq$ of his preferences, his budget $w>0$ and the vector $p \in \mathrm{R}_{+}^{\mathrm{n}}$ of the unit prices of the goods to be chosen inside the convex set of goods $G \subseteq \mathrm{R}_{+}^{\mathrm{n}}$. The set of optimal choices is the set $X(p, w) \subseteq G$ defined by

$$
x \in X(p, w) \Longleftrightarrow[\langle p, x\rangle \leq w \text { and } y \preceq x \forall y \in G \text { such that }\langle p, y\rangle \leq w] .
$$

It is clear that $X(\lambda p, \lambda w)=X(p, w)$ for all $\lambda>0$. In reason of a non satiation assumption on the preorder one has $\langle p, x\rangle=w$ for all $x \in X(p, w)$. Hence,

$$
[\langle p, y-x\rangle \leq 0 \Longrightarrow y \preceq x] \quad \text { and } \quad[\langle p, y-x\rangle<0 \Longrightarrow y \prec x]
$$

$X$ is called the demand map associated to the preorder. In what follows we assume $X(p, w)$ to be nonempty for all $w>0$ and $p \in \mathrm{R}_{+}^{\mathrm{n}}$.

Let us consider any finite family $\mathcal{F}_{k}=\left\{\left(x^{i}, p^{i}, w_{i}\right)\right\}_{i=0}^{k+1} \subseteq \operatorname{gph}(X)$ such that $\left(x_{0}, p_{0}, w_{0}\right)=\left(x_{k+1}, p_{k+1}, w_{k+1}\right)$. The transitivity of the preorder implies

$$
\max _{i=0, \cdots, k}\left\langle p^{i}, x^{i+1}-x^{i}\right\rangle \geq 0
$$


and when the maximum equals 0 all $\left\langle p^{i}, x^{i+1}-x^{i}\right\rangle$ are null, to be compared with the analogous property on the subdifferential of convex functions. This property says that the map $-X(p, 1)$ is cyclically pseudomonotone $[13,8]$, and it is known by economists under the names of Generalized Axiom of Revealed Preferences (GARP) and Strong Axiom of Revealed Preferences (GARP), there are a few minor differences between the different formulations.

In what follows, we discuss on approximations of the preorder which can be obtained from a finite number of observations $\left(x^{i}, p^{i}, w_{i}\right) \in \operatorname{gph}(X), i \in I$. For simplicity, we place this discussion in the case where there is $\bar{t}>0$ such that $\bar{t} e \in G \subseteq[0, \bar{t}]^{n}, e=(1,1, \cdots 1) \in \mathrm{R}^{\mathrm{n}}$, and where the preorder can be represented by one utility $\bar{u}$ such that $\bar{u}(t e)=t$ for all $t \in[0, \bar{t}]$. We consider two different types of approximations.

9.2 Approximations Based on the Afriat's Construction.

Let $(\lambda, \mu)$ be any optimal solution of the linear program $[1,12,16,14]$

$$
\min _{\lambda, \mu}\left[\langle a, \lambda\rangle+\langle b, \mu\rangle: \lambda_{i} \geq 1, \mu_{i} \geq 0, \mu_{j}-\mu_{i} \leq \lambda_{i}\left\langle p^{i}, x_{j}-x^{i}\right\rangle \forall i, j \in I\right]
$$

where $a, b$ are two given nonnegative vectors. The cyclic property of $-X$ on the set of observations turns out to be equivalent to the feasibility of the linear program. Next, let us construct the function

$$
\widetilde{u}(x)=\min _{i}\left[\mu_{i}+\lambda_{i}\left\langle p^{i}, x-x^{i}\right\rangle\right] \quad \forall x \in G
$$

The function $\widetilde{u}$ is piecewise linear concave by construction. In reason of the cyclic property $\widetilde{u}\left(x^{i}\right)=\lambda_{i}$ and $\lambda_{i} p^{i}$ is a supgradient of $\widetilde{u}$ at $x^{i}$. In addition, 
$\widetilde{u}\left(x^{i}\right) \leq \widetilde{u}\left(x^{j}\right)$ if and only if $x^{i} \preceq x^{j}$. The function $\widetilde{u}$ depends on the family $\mathcal{F}$, the vectors $a$ and $b$ and the couple $(\lambda, \mu)$ chosen among the optimal solutions of the linear program. It is necessary to proceed to a normalisation on the functions $\widetilde{u}$ in order to get valuable comparisons.

The function $k$ defined by $k(t)=\widetilde{u}(t e)$ for all $t \in[0, \bar{t}]$ is concave and increasing. Let us take $u=k^{-1} \circ \widetilde{u}$. Then $u(t e)=t$ for all $t \in[0, \bar{t}]$. By construction, for $i, j=0,1, \cdots, k$,

$$
\begin{gathered}
x^{i} \in \arg \max _{x}\left[u(x): x \in G,\left\langle p^{i}, x\right\rangle \leq w_{i}\right], \\
u\left(x^{i}\right) \leq u\left(x^{j}\right) \Longleftrightarrow x^{i} \preceq x^{j},
\end{gathered}
$$

but $u(x) \leq u(y)$ is not equivalent to $x \preceq y$.

It is important to recall that the normalized utility $u$ obtained via this method depends on the choice of the pair $(a, b)$ of two positive numbers in the linear program. Another pair provides another $u$ and therefore a different approximation of the preorder.

9.3 Sandwich Type Approximations.

Here, $[8,9]$, one constructs an increasing quasiconcave function $u$ such that $u(t e)=t$ for all $t \in[0, \bar{t}], u\left(x^{j}\right) \leq u\left(x^{i}\right)$ whenever $\left\langle p^{i}, x^{j}-x^{i}\right\rangle \leq 0$ and $t=u(t e) \leq u\left(x^{i}\right)$ whenever $\left\langle p^{i}, t e-x^{i}\right\rangle \leq 0$. The first step consists to determine the values of $u$ at points $x^{i}$. Set $u_{i}=u\left(x^{i}\right), i \in I$. It is necessary that

$$
x_{i}^{i}=\min _{j} x_{j}^{i} \leq \frac{\left\langle p^{i}, x^{i}\right\rangle}{\left\langle p^{i}, e\right\rangle} \leq u_{i} \leq \max _{j} x_{j}^{i},
$$


and $\left\langle p^{i}, x^{j}-x^{i}\right\rangle \leq 0 \Rightarrow u_{j} \leq u_{i}$.

The existence of such a vector $u$ is equivalent to the cyclic property.

The second step consists to extend $u$ to the whole set $G$ in order to get an increasing quasiconcave function with $u(t e)=t$ for all $t \in[0, \bar{t}]$ and for $i \in I$

$$
u_{i}=u\left(x^{i}\right) \quad \text { and } \quad x^{i} \in \arg \max _{x}\left[u(x): x \in G,\left\langle p^{i}, x\right\rangle \leq w_{i}\right] .
$$

Among all the functions $u$ responding to these conditions there are two, $u_{-}$ and $u_{+}$, such that $u_{-} \leq u \leq u_{+}$for all other functions $u$. This is the case for the normalized approximations $u$ obtained via the Afriat's construction and also for the normalized utility function $\bar{u}$ associated with the preorder $\preceq$ when this preorder exists.

The relations between the functions $u_{-}$and $u_{+}$and the preorder $\preceq$ are

$$
u_{+}(x) \leq u_{-}(y) \Longrightarrow x \preceq y \quad \text { and } \quad u_{+}(x)<u_{-}(y) \Longrightarrow x \prec y
$$

to be compared with (8).

9.4 The Revealed Preference Problem.

Let us consider one increasing sequence of families $\cdots \subseteq \mathcal{F}_{p} \subseteq \mathcal{F}_{p+1} \subseteq \cdots$. It is expected that, under one density condition, the sequence of normalized utility functions $\left\{u^{p}\right\}$ generated with the Afriat's process converges to the normalized utility function $\bar{u}$ associated with the preorder when it is exists. In the sandwich process, the sequence $\left\{u_{-}^{p}\right\}$ is nondecreasing, the sequence $\left\{u_{+}^{p}\right\}$ is nonincreasing and $u_{-}^{p} \leq \bar{u} \leq u_{+}^{p}$ for all $p$. It is expected that both sequences 
converge to $\bar{u}$ (think of the Riemann integration). The problem is that, given $X$ enjoying the cyclic property, we are not sure of the existence of a preorder and consequently of its associated normalized utility function $\bar{u}$.

The long standing problem of Revealed Preferences $[19,24]$ consists in seeking what conditions on a multivalued map $X$ ensure the existence of one utility function $u$ associated with the preferences. The case where $X$ is differentiable is treated in [7] and [18]. The general case is very hard. It is shown in [8], see also [15], that the cyclic monotonicity of the multivalued map $-X(., 1)$ together with its maximality are not sufficient to ensure the existence and the unicity of a preorder associated with the preferences. An exemple is provided in [8] where the limits of the sequences $\left\{u_{-}^{p}\right\}$ and $\left\{u_{+}^{p}\right\}$ are not the same, giving two different preorders associated with the same demand map $X$.

Despite the very disturbing example in [8], the Afriat's and sandwich approximations from a finite panel of observations bring valuable informations. The theory of convexifiability, least convexity and revealed preferences needs again much work.

\section{Conclusion}

In this paper we show that a lsc quasiconvex is well defined through the family of the support functions of its level sets. It is then possible to characterize these quasiconvex functions which are convex. An interpolation process is used to build a convex function whose $p$ level sets are given. Then, we show that a positively homogeneous quasiconvex function can be written as the minimum 
of two convex functions. It is proved that a function as smooth as the ratio of two positive numbers is not locally convexifiable at any point of the positive orthant. The paper ends with some considerations on the approximation of preferences by utility functions.

Acknowledgements The author is very grateful to the referees for their careful readings. Their corrections, comments and remarks have been precious, the paper has been improved greatly thanks to the help of the referees.

\section{References}

1. Afriat, S.N.: The Construction of a Utility Function from Expenditure Date, Int. Econom. Rev. 8-1, 67-77 (1967)

2. Bolte, J., Pauwels, E.: Curiosities and counterexamples in smooth convex optimization. https://hal.archives-ouvertes.fr/hal-02447733 (2020)

3. Crouzeix, J.-P.: Contributions à l'étude des fonctions quasiconvexes. Thèse de doctorat es sciences, Université de Clermont 2 (1977)

4. Crouzeix, J.-P.: Conditions for convexity of quasiconvex functions. Math. Oper. Res. $5(1), 349-352(1980)$

5. Crouzeix, J.-P.: About differentiability of order one of quasiconvex functions on $\mathrm{R}^{\mathrm{n}}$. J. Optim. Theory Appl. 36-3, 367-385 (1982)

6. Crouzeix, J.-P.: Some properties of Dini-derivatives of quasiconvex and pseudoconvex functions. In: Gianessi, F., Rapcsák, T., Komlosi, S. (eds), New Trends in Mathematical Programming. Kluwer Academic Publishers, 41-57 (1998)

7. Crouzeix, J.-P., Rapcsák, T.: Integrability of pseudomonotone differentiable maps and the revealed preference problem. Journal of Convex Anal. 12-2, 43-446 (2005)

8. Crouzeix, J.-P., Eberhard, A., Ralph, D.: (Convex) Level Sets Integration. J. Optim. Theory Appl. 171-3, 85-886 (2016) 
9. Crouzeix, J.-P., Keraghel, A., Rahmani, N.: Integration of pseudomonotone maps and the revealed preference problem. Optimization 60, 783-800 (2011)

10. Debreu, G.: Theory of value, an axiomatic analysis of economic equilibrium. New Haven and London, Yale University Press (1959)

11. Debreu, G.: Least concave utility functions. J. Math. Econom. 3, 121-129 (1976)

12. Diewert, E.: Afriat and revealed preference theory. Rev. Econom. Stud. 40, 419-426 (1973)

13. Eberhard, A., Crouzeix, J.-P.: Existence of Closed Graph, Maximal, Cyclic PseudoMonotone Relations and Revealed Preference Theory. J. Ind. Manag. Optim. 3-2, $232-255(2007)$

14. Eberhard, A., Schreider, S., Kocoska, L., Crouzeix, J.-P., Ralph, D.: Some new approximation results for utilities in revealed preferences theory. 18th World IMACS/MODSIM Congress, Cairns (2009)

15. Eberhard, A., Ralph, D., Crouzeix, J.-P.: A Constructive Proof of the Existence of a Utility in Revealed Preference Theory. Unpublished manuscript.

16. Fostel, A., Scarf, H.E., Todd, M.J.: Two proofs of Afriat's theorem. Exp. Notes, Econom. Theory 24, 211-219 (2004)

17. Jensen, J.L.: Sur les fonctions convexes et les inégalités entre les valeurs moyennes. Acta Math. 30, 175-193 (1906)

18. Hadjisavvas N., Penot, J.P.: Revisiting the problem of integrability in utility theory. Optimization 64-12, 2495-2509 (2015)

19. Houthakker, H.S.: Revealed preferences and the utility function. Economica 17, 159174. (1950)

20. Kannai, Y.: Approximation of convex preferences. J. Math. Econom. 1, 101-106 (1974)

21. Kannai, Y.: Concavifiability and constructions of concave utility functions, J. Math. Econom. 4, 1-56 (1976)

22. Newman, P.: Some properties of concave functions. J. Econom. Theory 1, 291-314 (1969)

23. Offenbach, J., Meilhac, H., Halevy, L.: Calchas, act.1, sc. 5. La belle Hélène, Paris (1864) 
24. Samuelson, P.A.: The problem of integrability in utility theory. Economica 17-68, $355-385(1950)$ 